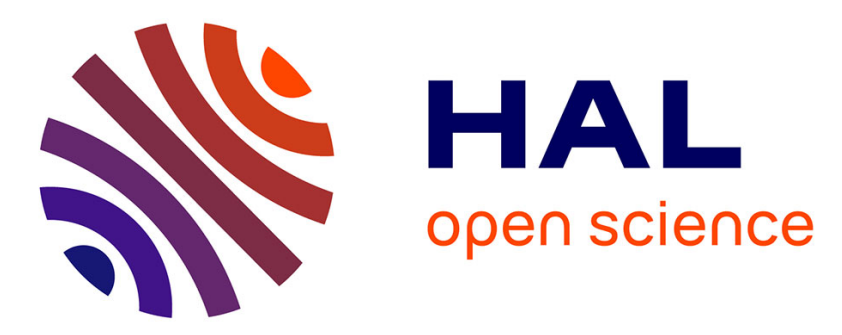

\title{
FES-Induced Muscular Torque Prediction with Evoked EMG Synthesized by NARX-Type Recurrent Neural Network
}

\author{
Zhan Li, Mitsuhiro Hayashibe, Qin Zhang, David Guiraud
}

\section{- To cite this version:}

Zhan Li, Mitsuhiro Hayashibe, Qin Zhang, David Guiraud. FES-Induced Muscular Torque Prediction with Evoked EMG Synthesized by NARX-Type Recurrent Neural Network. IROS: Intelligent Robots and Systems, Oct 2012, Vilamoura, Algarve, Portugal. pp.2198-2203, 10.1109/IROS.2012.6385602 . lirmm-00744448

\section{HAL Id: lirmm-00744448 \\ https://hal-lirmm.ccsd.cnrs.fr/lirmm-00744448}

Submitted on 23 Oct 2012

HAL is a multi-disciplinary open access archive for the deposit and dissemination of scientific research documents, whether they are published or not. The documents may come from teaching and research institutions in France or abroad, or from public or private research centers.
L'archive ouverte pluridisciplinaire HAL, est destinée au dépôt et à la diffusion de documents scientifiques de niveau recherche, publiés ou non, émanant des établissements d'enseignement et de recherche français ou étrangers, des laboratoires publics ou privés. 


\title{
FES-Induced Muscular Torque Prediction with Evoked EMG Synthesized by NARX-Type Recurrent Neural Network
}

\author{
Zhan Li, Mitsuhiro Hayashibe, Qin Zhang, and David Guiraud
}

\begin{abstract}
Functional electrical stimulation (FES) is able to restore motor function of spinal cord injured (SCI) patients. To make adaptive FES control taking into account the actual muscle state with muscular feedback information, torque estimation and prediction are important to be provided beforehand. Evoked EMG (eEMG) has been found to be highly correlated with FES-induced torque under various muscle conditions, indicating that it can be an useful tool for torque/force prediction. To better construct the relationship between eEMG and stimulated muscular torque, nonlinear-arx-type (NARXtype) model is preferred. This paper presents and exploits a NARX-type recurrent neural network (NARX-RNN) model for identification and prediction of FES-induced muscular dynamics with eEMG. Such NARX-RNN model is with a novel architecture for prediction, with robust prediction performance. To make fast convergence for identification of such NARXRNN, directly-learning pattern is exploited during the learning phase. Due to difficulty of choosing a proper forgetting factor of Kalman filter for predicting time-variant torque with eEMG, such NARX-RNN may be considered to be a better alternative as torque predictor. Data gathered from two SCI patients is used to evaluate the proposed NARX-RNN model. The NARX-RNN model shows promising estimation and prediction performance only based on eEMG.
\end{abstract}

\section{INTRODUCTION}

Functional electrical stimulation (FES) can be regarded as an effective technique to help spinal cord injured (SCI) patients restore their motor functions, since muscle contraction can be driven artificially by electrical stimulation pulses [1], [2]. Because of high nonlinearity, time variance or even uncertainty in muscle dynamics under FES, prediction of muscle behaviour can be thus complicated. For instance, muscle fatigue can drastically change the dynamics and the maximum available force while keeping the same set of stimulus parameters. To make better adaptive predictive control of muscle contraction with electrical stimulation, the actual force/torque of muscle is usually needed to be known in advance [3], [4]. Under electrical stimulation, eEMG was found to be highly correlated with FES-induced torque under various muscle conditions [5], [6], [7], [8], and this phenomenon was also found in the FES implanted SCI subject [9]. Moreover, eEMG signals are noninvasive and reliable capture of time variations of muscular state.

Z. Li, M. Hayashibe, and D. Guiraud are with both INRIA Sophia-Antipolis DEMAR team, and LIRMM CNRS/University of Montpellier, UMR 5506, 95 Rue de la Galéra, 34095 Montpellier cedex 5, France. Emails: zhan.li, mitsuhiro.hayashibe, david.guiraud $\}$ @inria.fr,lirmm.fr $\}$

Q. Zhang was with INRIA-LIRMM DEMAR team, and currently is with Department of Mechanical Systems Engineering, Tokyo University of Agriculture and Technology under JSPS postdoc program. Email: qin. zhangelirmm. fr
Comprehensively modeling both FES induction and eEMG can thus be feasible to estimate muscular contribution on the force production [10], [11]. Evidence has indicated that the relationship between eEMG and muscle force/torque is time-varying and highly nonlinear, which implies that staticparameter type estimation approaches [12] may be improper for identification.

To remedy current estimation/prediction methods for stimulated muscular force, a Kalman filter with fixed forgetting factor was proposed to estimate the muscular torque with FES induction and eEMG [13], by choosing Polynomial Hammerstein Model (PHM) with eEMG and introducing muscle torque [14] as the muscle-model input and output respectively. Such work shows better prediction results with the latest model estimation if the forgetting factor is well chosen. However, selecting the optimal fixed forgetting factor for such Kalman filter sometimes can be a tough task, otherwise oscillatory may occur during estimation and prediction (especially, without torque sensors providing feedback information). Owing to this, recurrent neural network (RNN) can be used as a wise approach for estimating nonlinear system [15], [16], making it as a promising tool for realtime mechanical and biomedical signal processing. In this paper, in order to improve performance of predicting potential muscle fatigue, a NARX-type recurrent neural network (NARX-RNN) model is proposed to establish the modeling between FES-induced torque/force and eEMG with real-time identification capability. Different from NARX model used in [13], the proposed NARX-RNN is established based on a NARX form supplemented with eEMG and torque coupled terms. To enhance convergence of the estimated parameters [16] of such NARX-RNN model, directly-learning pattern [17] is employed [18]. Based on the NARX-RNN model, the prediction results are presented on two SCI patients.

We organize the paper as follows. Together with the identification approach, the proposed NARX-RNN model for FES-induced the muscular torque dynamics with eEMG is introduced in detail at Sections II and III. In Sections IV, identification and prediction results based on data collected from two SCI subjects assess the efficiency of presented NARX-RNN approach, and comparison between NARXRNN and Kalman filter is addressed accordingly. Final conclusions and discussion are draw in Section V.

\section{NARX-RNN FOR FES-INDUCED MUSCLE MODELING WITH EVOKED-EMG}

Before preparing the identification with eEMG signals and measured torques, data processing was made by: 1) removing 


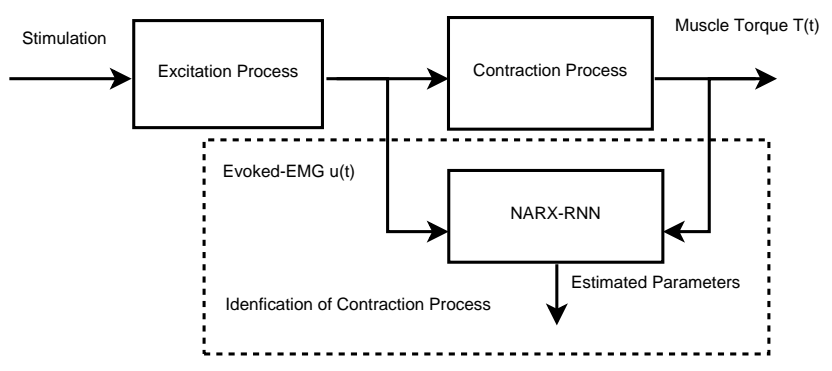

Fig. 1. NARX-RNN muscle model structure

stimulation artifacts; 2) applying low-pass filter for measured torque; 3 ) calculating the mean absolute value (MAV) of eEMG induced by FES. For more details on the stimulation experiment session and data processing procedure, please refer to [13]. The MAV of EMG $u(t) \in R$ and measured muscle torque $T(t) \in R$ are normalized with their maximum values respectively, before equipped into the model as the input and output targets for learning respectively, i.e., after normalization, $T(t)$ and $u(t)$ are within range $[0,1]$. At a given time instant $t$, we introduce the following NARXRNN model to represent the muscle contraction dynamics as follows.

$$
\begin{aligned}
T(t)= & \sum_{i=1}^{l} \sum_{j=1}^{m} w_{i j}(t) u^{j}(t-i)+\sum_{k=1}^{n} v_{k}(t) T(t-k) \\
& +a(t) u(t-l) T(t-1)+b(t) u(t-1) T(t-n),
\end{aligned}
$$

where coefficients $w(t)=\left[w_{11}(t), w_{12}(t), \cdots, w_{l m}(t)\right] \in R^{l m}$, $v(t)=\left[v_{1}(t), v_{2}(t), \cdots, v_{n}(t)\right] \in R^{n}, a(t) \in R$ and $b(t) \in R$ are parameters to be estimated with eEMG and measured torque within a period of time $t \in\left[0, t_{i d}\right]$. Corresponding to NARXRNN model (1), the structure of FES-induced muscle model with eEMG is as shown in Fig. 1. To ensure the performance of NARX-RNN model during prediction after identification off, proper model order $(l, m, n)$ can be thus important. In this paper, order $(l, m, n)=(5,3,4)$ is chosen for better prediction performance. The identified time-varying parameters for such NARX-RNN model (1) are trained and optimally obtained by direct learning pattern at time instant $t$. As time $t$ evolves, the parameters $w(t), v(t), a(t)$ and $b(t)$ are updated by training of NARX-RNN model (1) as well.

By exploiting directly-learning pattern [17] to estimate the NARX-RNN model (1) during time interval $t \in\left[0, t_{i d}\right]$, we can complete the training process and obtain the latest estimated parameters $w\left(t_{i d}\right) \in R^{l m}, v\left(t_{i d}\right) \in R^{n}, a\left(t_{i d}\right) \in R$ and $b\left(t_{i d}\right) \in R$. Since measured torque $T(t)$ can not be available if torque sensors are not equipped or with large-error measurement after time $t_{i d}$, the parameters aforementioned thus are not able to be updated. In this case, we can use the latest optimally-estimated parameters $w^{*}\left(t_{i d}\right) \in R^{l m}, v^{*}\left(t_{i d}\right) \in R^{n}$, $a^{*}\left(t_{i d}\right) \in R$ and $b^{*}\left(t_{i d}\right) \in R$ to make prediction after time $t_{i d}$, and the predicted torque $\hat{T}(t)$ is expressed as

$$
\begin{aligned}
& \hat{T}(t)=\sum_{i=1}^{l} \sum_{j=1}^{m} w_{i j}^{*}\left(t_{i d}\right) u^{j}(t-i)+\sum_{k=1}^{n} v_{k}^{*}\left(t_{i d}\right) \varphi(\hat{T}(t-k)) \\
& +a^{*}\left(t_{i d}\right) u(t-l) \varphi(\hat{T}(t-1))+b^{*}\left(t_{i d}\right) u(t-1) \varphi(\hat{T}(t-n)),
\end{aligned}
$$

with $t>t_{i d}$. Where $\varphi(\cdot)$ denotes nonnegative function described as

$$
\varphi(\hat{T}(t-k))= \begin{cases}\hat{T}(t-k), & \hat{T}(t-k) \geq 0 \\ 0, & \hat{T}(t-k) \leq 0\end{cases}
$$

here, we can emphasize that the torque is computed only based on the eEMG afterwards.

\section{NARX-RNN MODEL IDENTIFICATION}

To accelerate identification of the aforementioned NARXRNN model (1), in this section, we introduce and present the directly-learning pattern in order to identify parameters of NARX-RNN model (1). Such directly-learning or related methods in machine learning and neural network areas were already verified in simple nonlinear system identification issues [17]. In this work, we employ such learning method to identify parameters in NARX-RNN model (1) rather than use other traditional methods such as gradient-descent iterative methods [18].

During time interval of identification $t \in\left[0, t_{i d}\right]$, we have obtained and stored the measured torque $T(t)$ and eEMG signals $u(t)$. To identify NARX-RNN model (1), we firstly define the following batch square error function from $t=0$ to $t=t_{i d}$,

$$
\begin{aligned}
& E(t)=\frac{1}{t_{i d}} \sum_{t=0}^{t_{i d}}\left[T(t)-\sum_{i=1}^{l} \sum_{j=1}^{m} w_{i j}(t) u^{j}(t-i)-\sum_{k=1}^{n} v_{k}(t) T(t-k)\right. \\
& -a(t) u(t-l) T(t-1)-b(t) u(t-1) T(t-n)]^{2},
\end{aligned}
$$

or in matrix-vector form,

$$
E(t)=\frac{1}{t_{i d}}(T-D \theta)^{T}(T-D \theta),
$$

where torque vector is $T=\left[T(0), T(1), \cdots, T\left(t_{i d}\right)\right]^{T}$, parameters of NARX-RNN which are to be estimated are $\theta=\left[w_{11}\left(t_{i d}\right), \cdots, w_{l m}\left(t_{i d}\right), v_{1}\left(t_{i d}\right), \cdots, v_{n}\left(t_{i d}\right), a\left(t_{i d}\right), b\left(t_{i d}\right)\right]^{T}$, and matrix involving past eEMG and torque information is $D=\left[X_{1}, X_{2}, \cdots, X_{l}, Y, C_{1}, C_{2}\right]$. During the whole identification phase, matrices $T$ and $D$ are being stored as time goes.

Specifically, the matrices $X_{1}, \cdots, X_{l}, Y, C_{1}, C_{2}$ composing matrix $D$ can be written in detail respectively as follows.

$$
X_{1}=\left[\begin{array}{cccc}
0 & 0 & \cdots & 0 \\
u(0) & u^{2}(0) & \cdots & u^{m}(0) \\
\vdots & \vdots & \ddots & \vdots \\
u\left(t_{i d}-l\right) & u^{2}\left(t_{i d}-l\right) & \cdots & u^{m}\left(t_{i d}-l\right) \\
\vdots & \vdots & \ddots & \vdots \\
u\left(t_{i d}-1\right) & u^{2}\left(t_{i d}-1\right) & \cdots & u^{m}\left(t_{i d}-1\right)
\end{array}\right]
$$




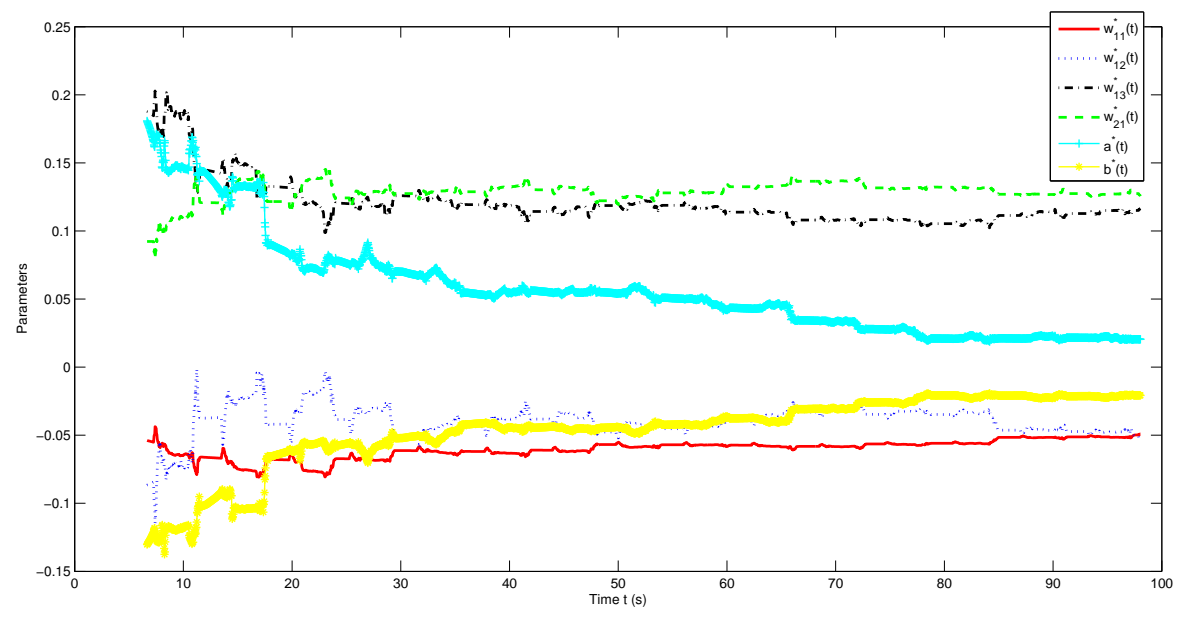

Fig. 2. Estimated time-varying parameters $\theta^{*}(t)$ of NARX-RNN model (1), plotted from time around $t=7 \mathrm{~s}$

$X_{l}=\left[\begin{array}{cccc}0 & 0 & \cdots & 0 \\ 0 & 0 & \cdots & 0 \\ \vdots & \vdots & \ddots & \vdots \\ 0 & 0 & \cdots & 0 \\ u(0) & u^{2}(0) & \cdots & u^{m}(0) \\ \vdots & \vdots & \ddots & \vdots \\ u\left(t_{i d}-l\right) & u^{2}\left(t_{i d}-l\right) & \cdots & u^{m}\left(t_{i d}-l\right)\end{array}\right]$,
$Y=\left[\begin{array}{cccc}0 & 0 & \cdots & 0 \\ T(0) & 0 & \cdots & 0 \\ T(1) & T(0) & \cdots & 0 \\ \vdots & \vdots & \ddots & \vdots \\ T\left(t_{i d}-1\right) & T\left(t_{i d}-2\right) & \cdots & T\left(t_{i d}-n\right)\end{array}\right]$,

$$
C_{1}=\left[\begin{array}{c}
0 \\
0 \\
\vdots \\
u(0) \\
\vdots \\
u\left(t_{i d}-l\right)
\end{array}\right] \circ\left[\begin{array}{c}
0 \\
T(0) \\
\vdots \\
T\left(t_{i d}-1\right)
\end{array}\right],
$$

and

$$
C_{2}=\left[\begin{array}{c}
0 \\
u(0) \\
\vdots \\
u\left(t_{i d}-1\right)
\end{array}\right] \circ\left[\begin{array}{c}
0 \\
0 \\
\vdots \\
T(0) \\
\vdots \\
T\left(t_{i d}-n\right)
\end{array}\right]
$$

where operator $\circ$ denotes Hadamard product or Schur product between vectors.

To obtain the optimal estimated parameters $\theta^{*}$ of NARXRNN model, the error function $E(t)$ has to be forced to zero during the learning process. By considering the gradient $\nabla E$ of error function with respect to $\theta$ using the directly-learning style [17], the optimal identified parameters $\theta^{*}$ is

$$
\theta^{*}=D^{\dagger} T
$$

where notation $D^{\dagger}$ is the pseudoinverse of matrix $D$. Such estimation equation provides a more straightforward way for getting the accurate parameters for identifying NARX-RNN models. From procedures above, we can observe that many zeros appear in the matrices and vectors, which implies that, computational time consumption for solving pseudoinverse of matrix $D$ can be rather low. That is to say, the time cost for identifying the parameters of NARX-RNN model (1) describing the relationship between FES-induced torque and eEMG can be low. This point is important for online predictive FES control of muscle for controller to establish the predictive model instantly after the identification phase.

As the torque sensors may be not always available in practice, to perform possible better predictive control of muscle activity, we need to predict the muscle torque only based on eEMG. In such case, real-time identification of muscle dynamics are switched off after time instant $t_{i d}$ and thus muscle model updating is stopped. We use the latest identified parameters $\theta^{*}$ till $t=t_{i d}$ to predict the muscle torque and measured torque will be never considered afterwards, i.e., according to equation (1), the predicted torque can be as follows.

$$
\begin{aligned}
& \hat{T}(t)=\theta^{* T}\left[u(t-1), \cdots, u^{m}(t-l), \varphi(\hat{T}(t-1)), \cdots,\right. \\
& \varphi(\hat{T}(t-n)), u(t-l) \varphi(\hat{T}(t-1)), u(t-1) \varphi(\hat{T}(t-n))]^{T} .
\end{aligned}
$$

It is worth noting here that, Kalman filter with forgetting factor for predicting muscular torque with estimated parameters has to be with a proper chosen (fixed) forgetting factor, which is usually within the range [0.9 1] [13]. Otherwise, such Kalman filter may encounter instability and unsatisfactory output results. Compared with Kalman filter with forgetting factor, the proposed NARX-RNN model with its 


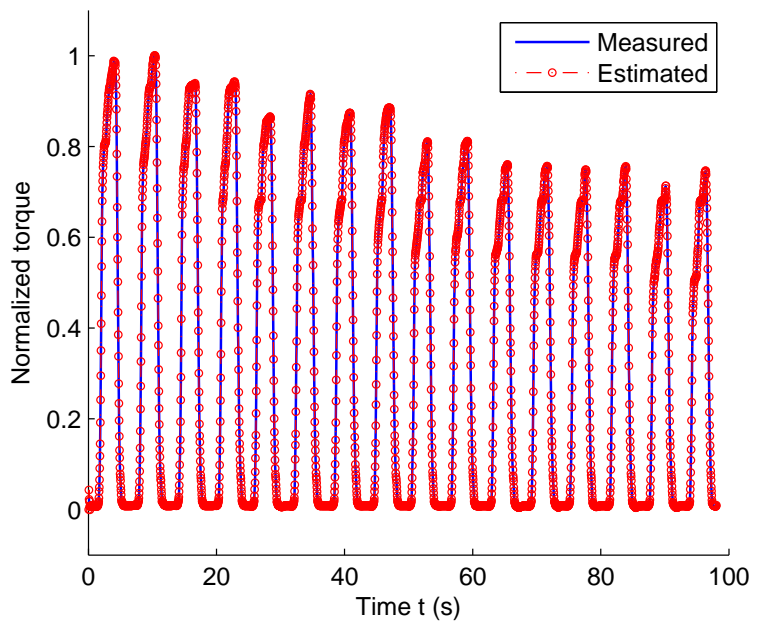

(a) One step identification of NARX-RNN model (1)

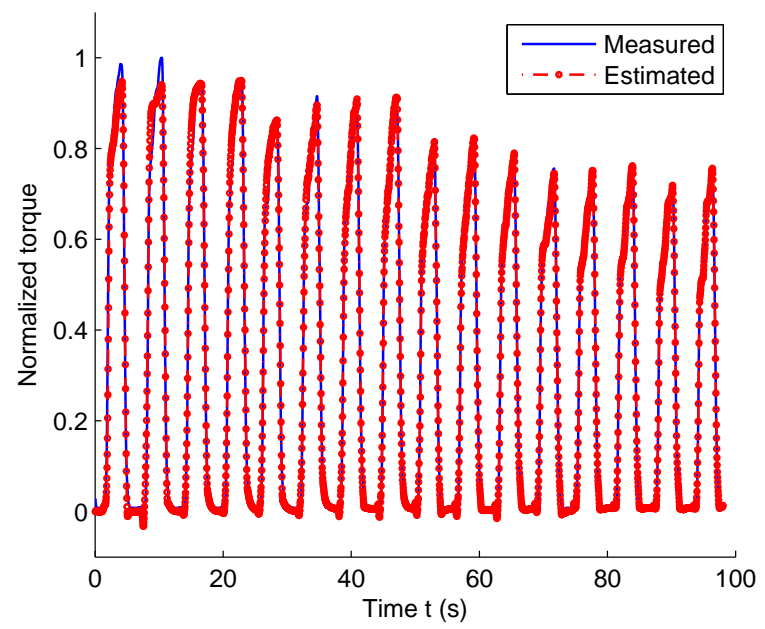

(b) Online identification of Kalman filter

Fig. 3. Performance of NARX-RNN model (1) and Kalman filter with forgetting factor for identification of FES-induced muscular torque with eEMG

directly-learning estimator, may be regarded as a more robust alternative.

\section{VERification BASEd on ExPERIMENTAL DATA}

In this section, we focus on verification of the proposed NARX-RNN model (1) via data from two SCI subjects. The dataset for testing the NARX-RNN model was collected from experiment performed in Propara Rehabilitation Center, Montpellier, France. The experiment was approved by the France ethical committee and all subjects signed informed consent forms. The patient configuration is shown by Tab. I [13]. T6 means that 6th thoracic vertebra is damaged for their injury. The experiment setup is described briefly as follows [13]. The muscle group was stimulated with amplitude modulation at a constant frequency $(30 \mathrm{~Hz})$ and constant pulse-width $(450 \mu \mathrm{s})$, under isometric conditions by a portable stimulator. The eEMG activity of soleus was recorded, amplified (gain 1000) and sampled at $4 \mathrm{kHz}$ by an acquisition system. The subjects were seated on the chair with their ankle at $90^{\circ}$, while the foot was strapped on the pedal. For purposes of comparison, Kalman filter with forgetting factor [13] is exploited for the muscular torque identification and prediction with eEMG, based on the NARX-type muscle model used in [13]. The identification results in subject P1 will be presented firstly, and then the prediction results only based on eEMG will be especially introduced and emphasized.

\section{A. NARX-RNN Model for Identification}

The identified parameter $\theta^{*}$ is actually a time-varying term determined by the measured muscle torque and eEMG signals at each latest time instant. Fig. 2 illustrates the identified parameter $\theta^{*}(t)$ from time $t=7 \mathrm{~s}$ around of subject $\mathrm{P} 1$. This figure shows that the muscle state is time-variant
TABLE I

Patient CONFigurations

\begin{tabular}{|c|c|c|c|c|c|}
\hline $\begin{array}{c}\text { Test } \\
\text { subject }\end{array}$ & $\begin{array}{c}\text { Age } \\
(\text { years })\end{array}$ & $\begin{array}{c}\text { Weight } \\
(\mathrm{kg})\end{array}$ & $\begin{array}{c}\text { Height } \\
(\mathrm{cm})\end{array}$ & $\begin{array}{c}\text { Level of } \\
\text { injury }\end{array}$ & $\begin{array}{c}\text { Months post } \\
\text { injury }\end{array}$ \\
\hline P1 & 26 & 64 & 192 & T6 & 36 \\
\hline P2 & 48 & 76 & 177 & T6 & 18 \\
\hline
\end{tabular}

with its identified parameters time-varying. The identification performance of NARX-RNN model (1) and Kalman filter in Subject P1 is shown by Fig. 3, with the latest identified parameter at the end time instant. Observed from Fig. 3, the performance of NARX-RNN model (1) for identification can be a little better than that of Kalman filter with forgetting factor $\lambda=0.997$. The identification, made by NARX-RNN model (1) can only need to estimate the latest parameter $\theta^{*}$ with global information of entire eEMG and measured torque, so we can call it is as the "one step" identification. It is worth pointing here that one difference between NARXRNN model (1) and Kalman filter may lie in that: NARXRNN model (1) requires sufficient amount of data (both of eEMG and measured FES-induced torque) to obtain the latest parameters and ensure the accuracy, which implies it is not a strictly online estimator at every time instant $t$; nevertheless Kalman filter with forgetting factor is a recursive estimator in nature updating its coefficients (except the fixed forgetting factor) using the last coefficients at every time instant $t$, which implies it is a strict online estimator. For realistic application, NARX-RNN model (1) provides one possibility to finish identification task at the end time instant by only one step with all eEMG and measured torque collected.

\section{B. NARX-RNN Model for Prediction Based on eEMG}

Joint torque measurement equipment can not be used in daily life of the patients, as normally such device is 

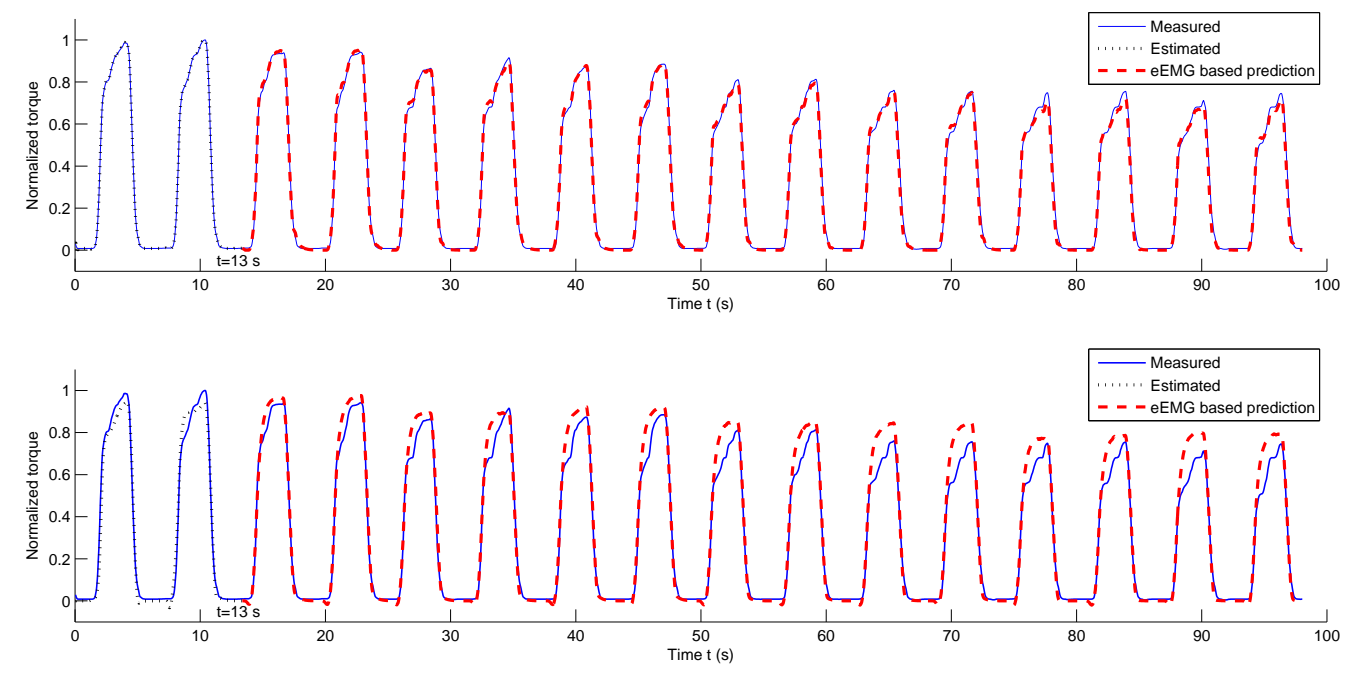

Fig. 4. Performance of NARX-RNN model and Kalman filter with forgetting factor for predicting muscular torque after $t=13 \mathrm{~s}$ with eEMG. Upper: Estimation and prediction synthesized by NARX-RNN. Lower: estimation and prediction is synthesized by Kalman filter with forgetting factor $\lambda=0.997$
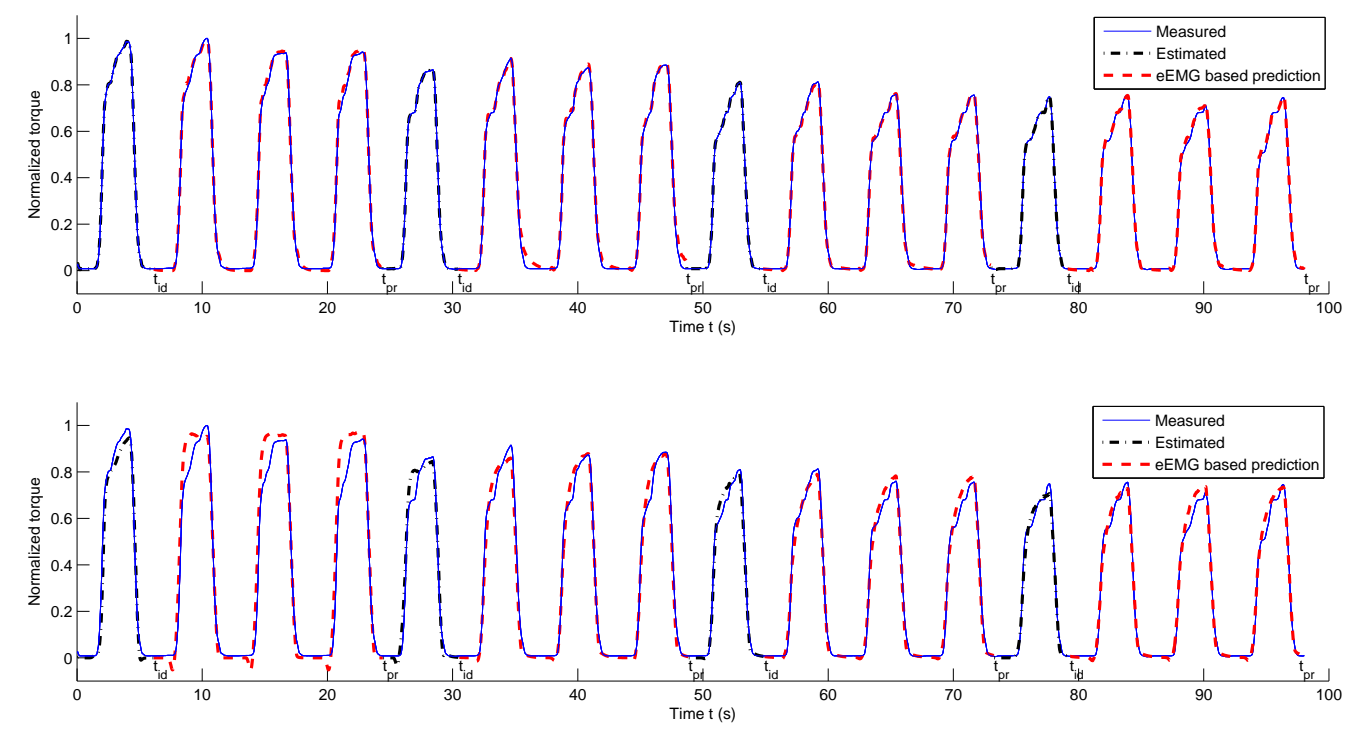

Fig. 5. Performance of NARX-RNN model and Kalman filter with forgetting factor with eEMG for prediction with identification on periodically. Upper: Estimation and prediction synthesized by NARX-RNN. Lower: estimation and prediction is synthesized by Kalman filter with forgetting factor $\lambda=0.997$

not portable and implanted sensor is not available. In this scenario, we show the performances of NARX-RNN model and Kalman filter in Subject P1 under such environment. Identification is completed by time instant $t=13 \mathrm{~s}$ and then switched off, and prediction is launched at $t=13 \mathrm{~s}$. From the time instant the joint torque estimated by NARX-RNN model is only driven by the eEMG signals. Seen from Fig. 4, obviously NARX-RNN model performs better than that of Kalman filter with forgetting factor $\lambda=0.997$ with less errors, and shows fairly a longer prediction horizon at the same error level. To investigate the prediction horizon of both two approaches, we stop identification by $6 \mathrm{~s}$ and make prediction from then without updating muscle models, evaluating the root mean square (RMS) errors at different prediction horizon 10s, 30s, 50s and 70s. As further illustrated by Tab. II, at the same length prediction horizon, RMS error of NARX-RNN model is lower than that of Kalmanfilter estimated muscle model for subjects P1 and P2. All of these may imply that NARX-RNN model can possess superior robustness on prediction of muscle torque with only eEMG signals. In addition, the model identified by Kalman filter with forgetting factor tends to have shorter prediction 
TABLE II

PREDICTION HORIZON COMPARISON WITH SUBJECTS P1 AND P2

\begin{tabular}{|c|c|c|c|c|c|}
\hline \multirow{2}{*}{ Subject } & \multirow{2}{*}{ RMS error } & \multicolumn{4}{|c|}{ Prediction horizon } \\
\cline { 3 - 6 } & & $10 \mathrm{~s}$ & $30 \mathrm{~s}$ & $50 \mathrm{~s}$ & $70 \mathrm{~s}$ \\
\hline \multirow{2}{*}{ P1 } & NARX-RNN & 0.0407 & 0.0530 & 0.0552 & 0.0587 \\
\cline { 2 - 6 } & Kalman filter & 0.0751 & 0.0860 & 0.0950 & 0.1067 \\
\hline \multirow{2}{*}{ P2 } & NARX-RNN & 0.0402 & 0.0520 & 0.0554 & 0.0598 \\
\cline { 2 - 6 } & Kalman filter & 0.0503 & 0.0631 & 0.0656 & 0.0663 \\
\hline
\end{tabular}

horizon on the two subjects at the same error level. The experimental data contains the torque effected by muscle fatigue inducing tests. In FES, the control issue under the muscle fatigue is a major problem. We definitely need the method to predict the time-variant muscle response based on biofeedback signals for longer prediction horizon. The NARX-RNN method shows promising performance in the preliminary test.

One can evaluate the prediction performance of NARXRNN model and Kalman filter with forgetting factor assuming the periodic events of missing torque measurement, and identification is switched off during these events. As in Fig. 5, identification is terminated at time instant $t_{i d}$ and then prediction is started afterwards with the latest estimated parameters from time instant $t_{i d}$ and ended at time instant $t_{p r}$. The process circle is repeated periodically. During the first time period $\left[\begin{array}{ll}t_{i d} & t_{p r}\end{array}\right]$, NARX-RNN model shows better prediction results than that of Kalman filter. After updating muscle model in the future circles, the prediction performance of Kalman filter improves. However, observed from Fig. 5, NARX-RNN produces better prediction results than that of Kalman filter in the whole experiment process. This implies NARX-RNN model can give also recursive and adaptive way of identification which is the known advantage in Kalman filter. Thus, the proposed method may have interesting features of identification where the prediction accuracy is maintained keeping its adaptive tracking capabilities. As for the computational cost, the average time of each single identification circle of NARX-RNN is around 0.0046s in MATLAB running environment, and that of Kalman filter is about $0.0298 \mathrm{~s}$. The main reason for the difference may lie in two factors: 1) The NARX model architecture which NARX-RNN possesses is with eEMG-torque-coupled information additionally; 2) NARX-RNN exploited entire history of information of eEMG and torque to identify optimal parameters at the end time instant of identification phase, but Kalman filter uses such information locally in a recursive manner.

\section{CONClusions And Future Work}

A NARX-RNN-type muscle model has been proposed for identification and prediction for FES-induced muscular dynamics with eEMG. Such NARX-RNN is with directlylearning pattern which guarantees its promising estimation and lower computational cost. Experiment data obtained the from two SCI subjects is applied to verify the proposed NARX-RNN model. For purposes of comparison, Kalman filter with forgetting factor is also exploited with NARX muscle model addressed in [13] for the torque estimation on the same two subjects. The NARX-RNN model shows better identification performance. Especially, when the joint torque should be estimated only based on eEMG, eEMG driven prediction synthesized by the NARX-RNN model produces effective results, which demonstrate a longer prediction horizon on the two subjects. During muscle fatigue under FES, the predicted torque can be further used for adaptive closedloop FES control as compensation of muscle fatigue. Future work can be extended to adaptive closed-loop FES control for dynamic motion based on eEMG sensing with use of joint angle sensors.

\section{REFERENCES}

[1] A. Kralj and T. Bajd, Functional Electrical Stimulation: Standing and Walking after Spinal Cord Injury. CRC, Boca Raton, FL, 1989.

[2] D. Guiraud, T. Stieglitz, K. P Koch, J. Divoux, and P. Rabischong, "An impantable neuroprosthesis for standing and walking in paraplegia: 5 year patient follow-up," J. Neural Eng., vol. 3, pp. 268-275, 2006.

[3] M. Hayashibe, Q. Zhang, and C. Azevedo-Coste, "Dual predictive control of electrically stimulated muscle using biofeedback for drop foot correction," in Proc. IEEE/RSJ Int. Conf. Intell. Robots Syst., San Francisco, U.S.A., 2011. pp. 1731-1736.

[4] J. Ding, A. S. Wexler, and S. A. Binder-Macleod, "A predictive model of fatigue in human skeletal muscles," J. Appl. Physiol., vol. 89, no. 4, pp. 1322-1332, 2000.

[5] N. C. Chesler and W. K. Durfee, "Surface EMG as a fatigue indicator during FES-induced isometric muscle contractions," J. Electromyography Kinesiol., vol. 7, no. 1, pp. 27-37, 1997.

[6] A. Erfanian, H. J. Chizeck, and R. M. Hashemi, "Excitation contraction fatigue during sustained electrical stimulation of paralyzed muscle," in Proc. 18th Annu. Int. Conf. IEEE Eng. Med. Biol. Soc., Amsterdam, The Netherlands, 1996, pp. 1460-1461.

[7] Q. Shao, D. N. Bassett, K. Manal, T. S. Buchanan, "An EMG-driven model to estimate muscle forces and joint moments in stroke patients," Comput. Biol. Med., vol.39, pp. 1083-1088, 2009.

[8] A. Erfanian, H. J. Chizeck, and R. M. Hashemi, "Using evoked EMG as a synthetic force sensor of isometric electrically stimulated muscle," IEEE Trans. Biomed. Eng., vol. 45, no. 2, pp. 188-202, Feb. 1998.

[9] M. Hayashibe, Q. Zhang, D. Guiraud, and C. Fattal, "Evoked EMGbased torque prediction under muscle fatigue in implanted neural stimulation," J. Neural Eng., vol. 8, pp. 064001, 2011.

[10] J. Mizrahi, D. Seelenfreund, E. Isakov, and Z. Susak, "Predicted and measured muscle forces after recoveries of differing durations following fatigue in functional electrical stimulation," Artif. Organs., vol. 21, no. 3, pp. 236-239, 1997.

[11] J. Mizrahi, M. Levy, H. Ring, E. Isakov, and A. Liberson, "EMG as an indicator of fatigue in isometrically FES-activated paralyzed muscles," IEEE Trans. Rehabil. Eng., vol. 2, no. 2, pp. 57-65, Jun. 1994.

[12] T. L. Chia, P. Chow, and H. J. Chizeck, "Recursive parameter identification of constrained systems: An application to electrically stimulated muscle," IEEE Trans. Biomed. Eng., vol. 38, no. 5, pp. 429-442, May 1991.

[13] Q. Zhang, M. Hayashibe, P. Fraisse, and D. Guiraud, "FES-induced torque prediction with evoked emg sensing for muscle fatigue tracking," IEEE/ASME Trans. Mech., vol. 16, no. 5, pp. 816-826, Oct. 2011.

[14] E. J. Dempsey and D. T. Westwick, "Identification of Hammerstein models with cubic spline nonlinearities," IEEE Trans. Biomed. Eng., vol. 51, no. 2, pp. 237-245, Feb. 2004.

[15] D. Mandic and J. Chambers, Recurrent Neural Networks for Prediction: Learning Algorithms, Architectures and Stability. London: Wiley, 2001

[16] D. Wang, K. Lum, and G. Yang, "Parameter estimation of ARX/NARX model: a neural network based method," in Proc. 9th Int. Conf. Neural Inform. Processing, vol. 3, 2002, pp. 1109-1113.

[17] C. M. Bishop, Pattern Recognition and Machine Learning. New York: Springer, 2006.

[18] J. Luh, G. Chang, C. Cheng, J. Lai, S. Kuo, "Isokinetic elbow joint torques estimation from surface EMG and joint kinematic data: using an artificial neural network model," J. Electr. Kines., vol. 9. pp. 173183, 1999. 